\title{
Novel IRF6 mutations in Honduran Van Der Woude syndrome patients
}

\author{
ANDREW C. BIRKELAND ${ }^{1 *}$, YUNA LARRABEE ${ }^{*}$, DAVID T. KENT $^{7}$, CARLOS FLORES $^{8}$, \\ GLORIA H. SU ${ }^{2,3}$, JOSEPH H. LEE ${ }^{4,6}$ and JOSEPH HADDAD Jr ${ }^{2,5}$
}

\begin{abstract}
${ }^{1}$ Columbia University College of Physicians and Surgeons; Departments of ${ }^{2}$ Otolaryngology/Head and Neck Surgery,
${ }^{3}$ Pathology, ${ }^{4}$ Epidemiology and ${ }^{5}$ Pediatric Otolaryngology; ${ }^{6}$ Gertrude H Sergievsky Center and Taub Institute,

Columbia University Medical Center, New York, NY; ${ }^{7}$ Department of Otolaryngology, University of Pittsburgh Medical Center,

Pittsburgh, PA, USA; ${ }^{8}$ Department of Plastic Surgery, Hospital Escuela, University of Honduras, Tegucigalpa, Honduras
\end{abstract}

Received October 26, 2010; Accepted December 29, 2010

DOI: $10.3892 / \mathrm{mmr} .2011 .423$

\begin{abstract}
Van der Woude syndrome (VWS) is an autosomal dominant inherited disease characterized by lower lip pits, cleft lip and/or cleft palate. Missense, nonsense and frameshift mutations in IRF6 have been revealed to be responsible for VWS in European, Asian, North American and Brazilian populations. However, the mutations responsible for VWS have not been studied in Central American populations. Here, we investigated the role of IRF6 in patients with VWS in a previously unstudied Honduran population. IRF6 mutations were identified in four out of five VWS families examined, which strongly suggests that mutations in IRF6 are responsible for VWS in this population. We reported three novel mutations and one previously described mutation. In the first family, a mother and daughter both exhibited a p.N88I mutation in the DNA-binding region of IRFG that was not present in unaffected family members. In the second, we found a unique p.K101QfsX15 mutation in the affected patient, leading to a frameshift and early stop codon. In the third, we identified a p.Q208X mutation occurring in exon 6. In the fourth, we found a nonsense mutation in exon 9 (p.R412X), previously described in Brazilian and Northern European populations. In the fifth, we did not identify any unique exonic missense, nonsense or frameshift mutations. This study reports the first cases of IRF6 mutations in VWS patients in a Central American population, further confirming that the causal link between IRF6 and VWS is consistent across multiple populations.
\end{abstract}

Correspondence to: Dr Joseph Haddad Jr, Department of Pediatric Otolaryngology, Columbia University Medical Center, 3959 Broadway, Suite 501N, NY 10032, USA

E-mail: jh56@columbia.edu

${ }^{*}$ Contributed equally

Key words: Van der Woude syndrome, IRF6, Honduras, novel mutations

\section{Introduction}

Cleft lip with or without cleft palate $(\mathrm{CL} / \mathrm{P})$ is a common congenital malformation, presenting in 1/500 to 1/2000 births, with increased prevalence in Hispanic, Native American and Chinese populations. CL/P occurs in non-syndromic or syndromic forms, with non-syndromic forms constituting the majority $(\sim 70 \%)$ of cases. Of the syndromic forms of CL/P, Van der Woude syndrome (VWS; OMIM 119300) is the most common. It is responsible for $2 \%$ of cases of clefting overall, with an incidence of $1 / 35,000$ to $1 / 100,000$ births (1). VWS is an autosomal dominant disease with greater than $90 \%$ penetrance, characterized by lower lip pits, cleft lip, cleft palate and hypodontia (2). Popliteal pterygium syndrome (PPS; OMIM 119500) is a related disorder and is characterized by cleft lip, cleft palate, lower lip pits, syndactyly, pterygia in popliteal fossa and digital and genital anomalies, among other malformations (3). Families have been reported to have members expressing both VWS and PPS (4), suggesting that these syndromes may exist as a spectrum. Kondo et al (5) found that missense, nonsense and frameshift mutations in IRF6 were causal for VWS and PPS in North American, European and Brazilian patients. Since then, there have been further validating reports of IRF6 mutations in VWS in Asian, European and Brazilian populations $(4,6,7)$. In addition, single nucleotide polymorphisms in and around IRF6 have also been revealed to be associated with non-syndromic forms of CL/P (8), highlighting the importance of this gene in orofacial development.

The function of the IRF6 gene is poorly understood. It is part of the interferon regulatory factor family, which consists of 9 members. While other members are involved in infection response pathways, IRF6 does not share this role. IRF6 contains 10 exons and codes for a purported transcription factor, with exons 3-9 being the coding exons. Exons 3 and 4 constitute a tryptophan pentad repeat DNA-binding domain, while exons 7 and 8 form a Smad-interferon regulatory factorbinding domain. The DNA-binding domain, and to a lesser extent the protein-binding domain, are highly conserved.

We have previously reported an association between IRF6 and non-syndromic $\mathrm{CL} / \mathrm{P}$ in a Honduran population (9). Since previous studies in other populations demonstrated 

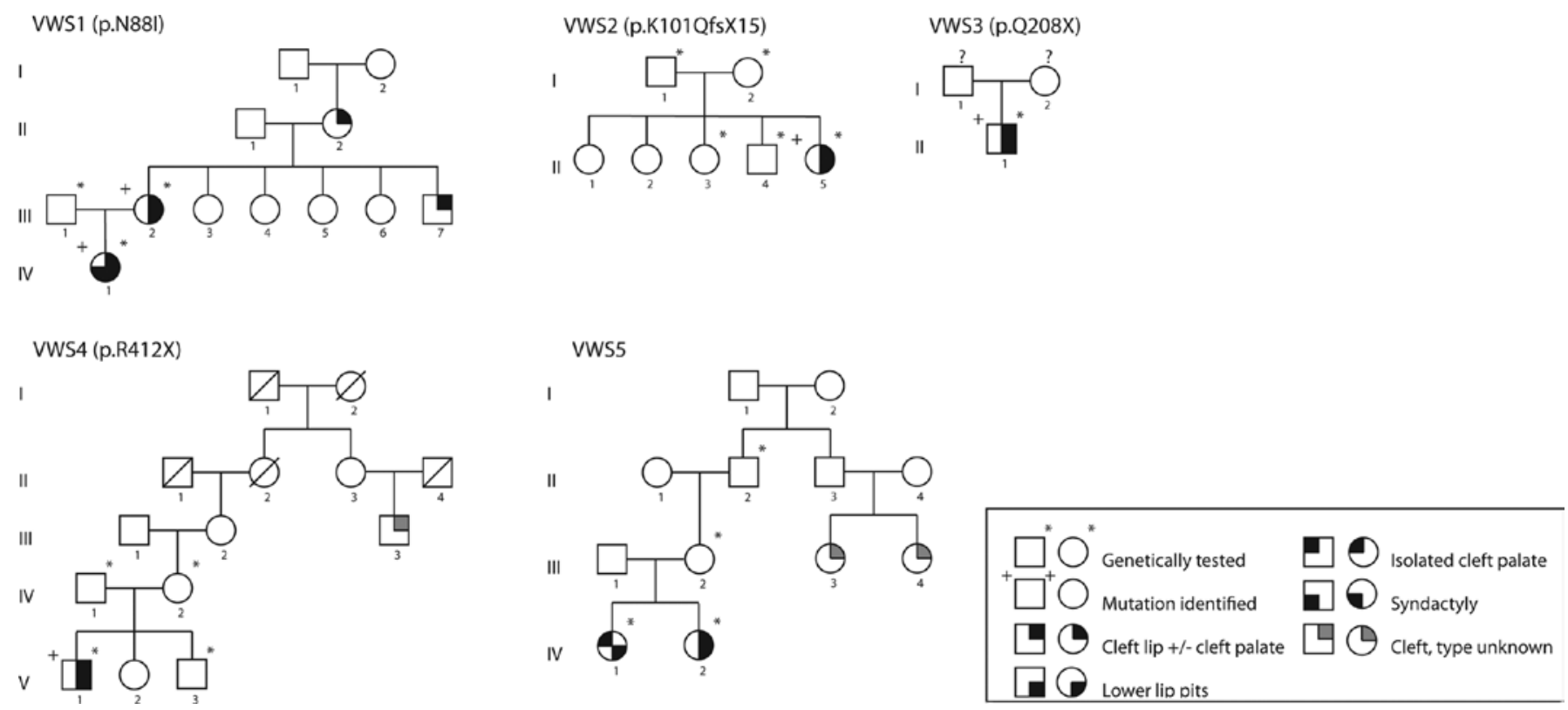

Figure 1. Family pedigrees of VWS patients. VWS1 reveals an autosomal dominant inheritance pattern. VWS2 reveals a de novo mutation in the patient, with no family history. VWS3 has an unknown family history. VWS4 reveals a de novo mutation in the patient and a distant relative exhibiting unknown cleft type. VWS5 reveals siblings with VWS. Notably, the mother's uncle has two daughters with clefts of unknown type.

that mutations in IRF6 were associated with VWS, here we examined a potential pleiotropic effect of IRF6 by extending our genetic study of clefting and IRF6 to VWS in a Honduran population.

\section{Materials and methods}

Patients. The study was approved by the Columbia University Medical Center Institutional Review Board. We screened for patients with VWS in a pediatric cleft clinic affiliated with the Honduran Medical Institute and the Department of Plastic Surgery of the Hospital Escuela, Tegucigalpa, Honduras. The presence of VWS was verified by oropharyngeal inspection and identification of clefting and lower lip pits. Complete histories were obtained, physical examination was performed, and patients were screened to avoid environmental confounders, such as folate antagonists. When available, family members were also examined for VWS and PPS phenotypes. Family histories and pedigrees were constructed based on patient reports.

Control subjects (100) were selected from a pediatric population recruited from the emergency, orthopedic, plastic surgery and general surgery departments of the Hospital Escuela. Control subjects were screened for the absence of a family history of $\mathrm{CL} / \mathrm{P}$ or other congenital anomalies.

Written informed consent was obtained from VWS patients and family members and the controls. Assent was obtained when applicable.

DNA samples. Venous blood was collected from patients with VWS and from available family members. Samples were shipped by overnight mail to the Columbia University Medical Center. Genomic DNA was isolated using the FlexiGene DNA-isolation kit (Qiagen, Valencia, CA, USA) per the manufacturer's protocol.
Mutation screening. Mutational analyses were performed using direct genomic sequencing. Primers were designed to cover the full exon and intron/exon boundaries for each exon individually, for exons 1-9 in IRF6. Primer sequences are available upon request. Polymerase chain reactions were performed to amplify each of the 9 exons individually from genomic DNA. Amplified DNA was then sent for DNA sequencing (Macrogen, Rockville, MD, USA). Sequences were analyzed using Sequence Scanner software (ABI, Foster City, CA, USA) and compared to consensus sequences using an NCBI BLAST search (http://blast.ncbi.nlm.nih.gov). We first sequenced all affected individuals to identify mutations and then genotyped all identified mutations in participating relatives and in controls. Sequences from patients with VWS were compared to control patient sequences to identify and exclude common population variants in IRF6. For all novel alterations, independent PCRs using both forward and reverse sequencing were performed to confirm a true mutation.

\section{Results}

Van der Woude syndrome families. Five families with VWS were identified from those attending the Honduran pediatric cleft clinic. One family (VWS1) had a history of three generations of CL/P, suggesting an autosomal dominant inheritance pattern (Fig. 1). Three other families (VWS2, VWS4 and VWS5) had no history of VWS in other generations, and physical inspection of available family members did not reveal signs of VWS (Fig. 1). One family (VWS5) consisted of a sibling pair affected by VWS with unaffected parents. One family (VWS3) consisted of a male patient, for whom family history was not obtained. An image of this patient's lower lip pits is depicted in Fig. 2. Pedigrees listing specific phenotypes are shown in Fig. 1. 


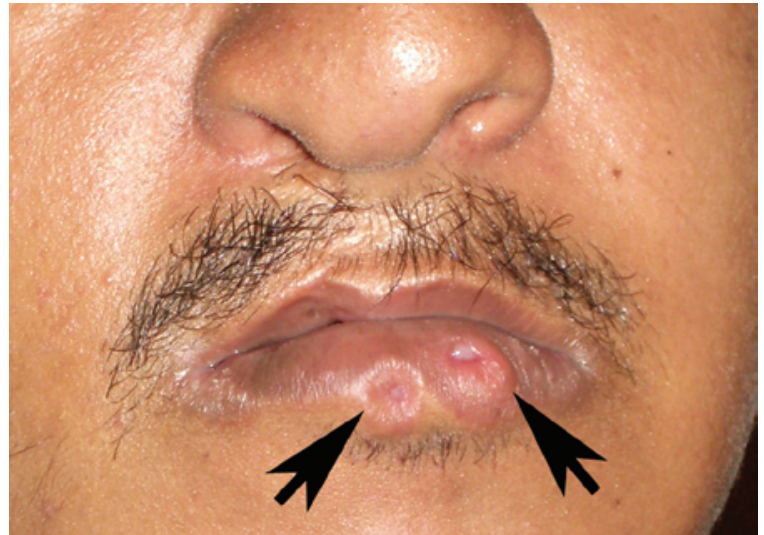

Figure 2. Patient with VWS from family VWS3 exhibiting lower lip pits (arrows) and repaired right cleft lip.

IRF6 mutations. Overall, four exonic IRF6 mutations in five families (a total of 5/7 affected individuals) were identified. The four identified mutations consisted of missense, nonsense and frameshift mutations (Table I) and were absent in unaffected family members. Three of the four mutations (p.N88I,
p.K101QfsX15 and p.Q208X) are novel and have not been reported previously.

A novel c.263A $>\mathrm{T}$ mutation causing an asparagine to isoleucine missense mutation was found in exon 4 (p.N88I) in both an affected mother and daughter (VWS1; Fig. 3A). A novel single base pair duplication mutation was identified (c.300dup C) in exon 4 of another proband (VWS2; Fig. 3A), resulting in a frame shift and an early stop codon 15 amino acids downstream in exon 4 (p.K101QfsX15). A novel c.622C > T mutation leading to a nonsense mutation (p.Q208X) was confirmed in exon 6 (VWS3; Fig. 3A). We also identified a c.1234C $>\mathrm{T}$ mutation leading to p.R412X, a nonsense mutation in exon 9 (VWS4; Fig. 3A) that has been previously described in Brazilian and Northern European populations (6). No unique exonic missense, nonsense or frameshift mutations were found in our fifth family (VWS5), although a c.921C>T mutation resulting in a conserved serine to serine mutation was found in exon 7 in the affected sibling pair (p.S307S). The unaffected mother had the same mutation.

Control population samples. To determine the frequencies of these mutations in the general Honduran population, 100 control subjects were sequenced. None of the unique

Table I. Exonic IRF6 mutations in VWS families

\begin{tabular}{llcc}
\hline Family & Mutation type & nt change & aa change \\
\hline VWS1 & Missense & c.263A $>$ T & p.N88I \\
VWS2 & Frameshift & c.300dup C & p.K101QfsX15 \\
VWS3 & Nonsense & c.622C $>$ T & p.Q208X \\
VWS4 & Nonsense & c.1234C $>$ T & p.R412X \\
VWS5 & Conserved & c.921C $>$ T & p.S307S \\
\hline
\end{tabular}

A

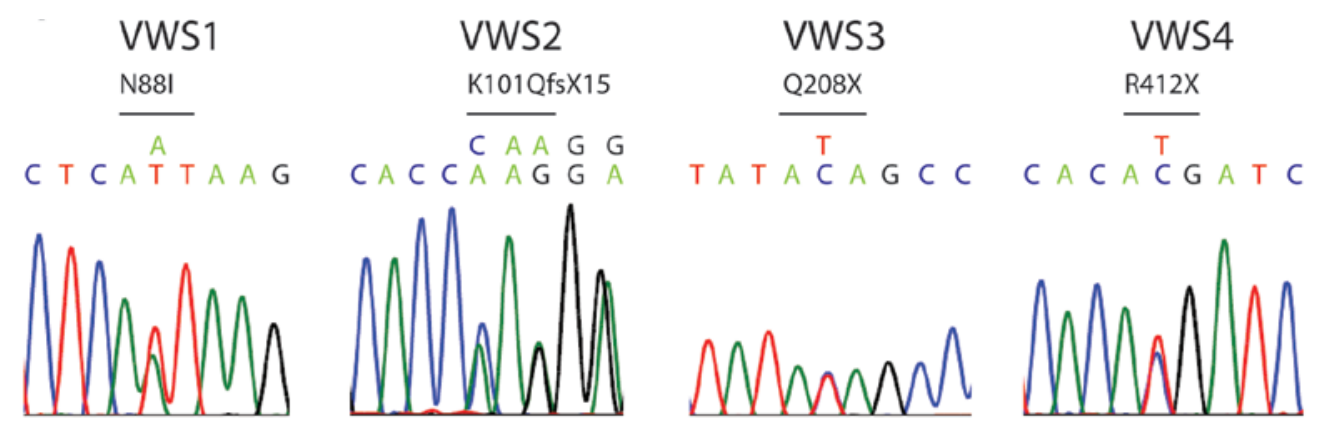

B

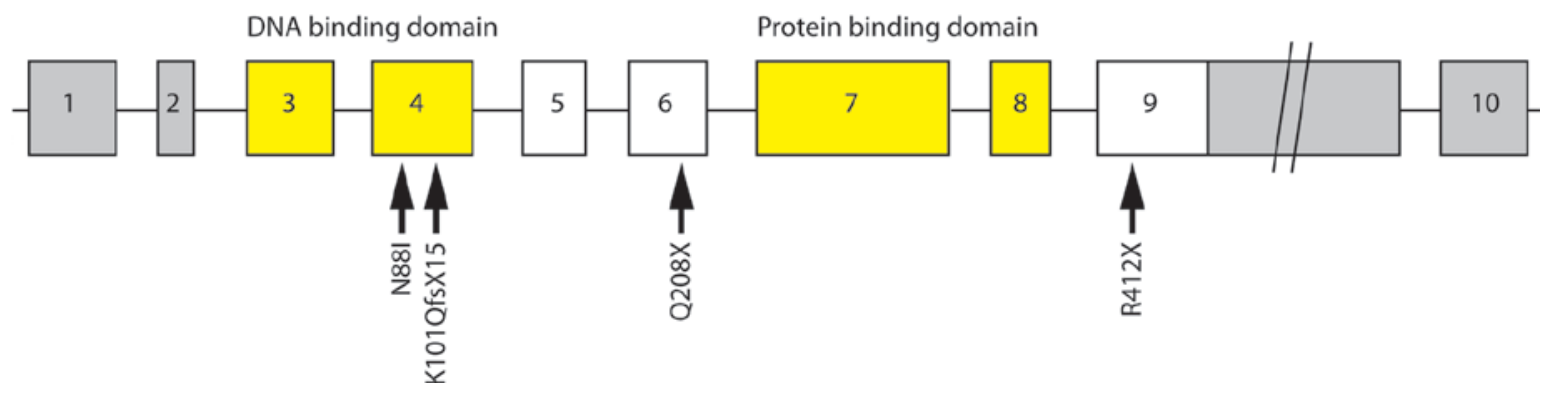

Figure 3. (A) Chromatogram sequences of mutations in IRF6. VWS1, Asn (AAT) to Ile (ATT) in exon 4; VWS2, C duplication leading to Lys (AAG) to Gln (CAA) frameshift in exon 4; VWS3, Gln (CAG) to Stop (TAG) in exon 6; VWS4, Arg (CGA) to stop (TGA) in exon 9. (B) Diagram of the IRF6 gene revealing mutation locations. Non-coding exons are in gray; DNA and protein binding domains are in yellow. Figure is drawn to scale except for exon 9 , which is truncated. 
mutations were identified in the control subjects. The previously described c.820G $>$ A polymorphism (causing a p.V274I change) (8) was found in control subjects with a minor allele frequency of 0.37 . This polymorphism was not found in any of the patients with VWS. No other unique missense, nonsense or frameshift mutations were found in the control patients.

\section{Discussion}

This is the first study to describe genetic mutations in VWS in a Central American population. Overall, we found four unique mutations in IRF6 in five patients, with three novel mutations that have not previously been reported. In each case, one copy of IRF6 was mutated and the other was normal, consistent with a haploinsufficiency model.

A unique mutation (p.N88I) was found in exon 4 of our first family (VWS1). The asparagine to isoleucine change is from a polar to non-polar amino acid. Using a protein structure damage predictor (Polyphen), this p.N88I mutation is thought to be damaging to the IRF6 protein structure. Asparagine 88 to histidine, tyrosine and serine mutations were previously described in VWS patients $(5,6)$, further suggesting that this is an important conserved residue. Mutations at this site may alter IRF6 gene function, likely through DNA-binding ability. The syndactyly in the affected daughter is consistent with previous reports that mutations in the DNA-binding region of IRF6 (exons 3 and 4) are associated with an increased likelihood of PPS findings (6), and that PPS and VWS findings are found in the same lineage (4).

The second pedigree (VWS2) contained an individual with a de novo insertion in exon 4 of IRF6, leading to a frame shift resulting in an early stop codon within exon 4 . Insertion mutations in IRF6 causing VWS have previously been described (6), but are less common than missense mutations. This mutation leads to both a disruption of the DNA-binding domain (exon 4) and to a complete loss of the protein-binding domain (exons 7 and 8).

A unique p.Q208X nonsense mutation was identified in exon 6 of the third patient (VWS3). This mutation is in a linker region between the DNA-binding and protein-binding domains of IRF6 (Fig. 3B). It is a less common region for mutations in VWS, although cases in other populations have been described (6). This early stop codon prevents the transcription of the protein-binding domain, and may alter the final structure of the IRF6 protein.

An arginine to stop mutation was found in exon 9 in the patient from the VWS4 family. The patient's mother, father and sibling all had normal IRF6 sequences. De Lima et al (6) found multiple individuals with VWS with p.Arg412X mutations in Northern European and Brazilian populations. The p.R412X mutation is thought to be a 'hotspot' mutation because it contains a $\mathrm{CpG}$ dinucletotide that is easily methylated to a $\mathrm{T}(10)$, as in this case, where it converted arginine (CAG) into a stop (TAG) codon.

A c. $921 \mathrm{C}>\mathrm{T}$ mutation leading to a conserved serine residue was found in exon 7 in a sibling pair from the VWS5 family. The unaffected mother had the same mutation, suggesting that this is not the causal mutation for VWS. No other mutations were identified in the exons of these patients. It has been suggested that approximately $68 \%$ of mutations in IRF6 causing VWS are in exonic regions (6), with the remainder likely in non-exonic IRF6 regions. The lack of a causative exonic mutation in this sibling pair suggests an unidentified non-exonic mutation in IRF6, or possibly a mutation in another gene. Interestingly, the mother's uncle had two daughters with clefting of unknown type (Fig. 2, VWS5 III-3 and III-4), suggesting a family predisposition to clefting with other contributing variants.

In all families, the exons and bordering introns of available relatives of patients with VWS were sequenced. The results revealed that family members did not carry the mutations in IRF6, further supporting that these are indeed the causal mutations for VWS. A total of 100 control patients were sequenced, none of whom carried unique missense, nonsense, or frameshift mutations in IRF6, further supporting previous reports that mutations in IRF6 are integral to the presence of VWS.

Much remains to be discovered in regards to the function of IRF6. It is thought to act as a transcription factor due to the presence of a DNA-binding domain and the observation that other members of the IRF family function as transcription factors (6). Mice deficient in IRF6 reveal a defect in keratinocyte proliferation and differentiation, leading to abnormal skin, limb and craniofacial development, including CL/P and PPS features $(11,12)$. The transcription factors $\mathrm{p} 63$ and AP- $2 \alpha$ have been revealed to be upstream regulators of IRF6 expression $(13,14)$, and mutations in these genes lead to syndromes involving $\mathrm{CL} / \mathrm{P}$, suggesting a common pathway $(15,16)$. Despite these recent advances, further studies are necessary to establish the function of IRF6.

A recent study in India did not find exonic IRF6 mutations in a 13-patient cohort, and suggested that IRF6 may not be responsible for VWS in a mixed Indian population (17), highlighting that various genes may be involved in different populations. Moreover, past groups have reported that the alternate chromosomal regions 1p34 (18) and 17p11 (19) may be associated with VWS. Thus, the verification of mutations in IRF6 in VWS in a previously unstudied Central American population was crucial. Here we identified mutations in IRF6 in patients with VWS in a population from Honduras, further strengthening results from other populations indicating that IRF6 mutations cause VWS.

\section{Acknowledgements}

The authors would like to thank Jose Arturo Pacheco Nunez for his assistance in identifying the families and collecting blood samples. This study was supported by The Honduran Medical Institute and the Doris Duke Charitable Foundation.

\section{References}

1. Gorlin RJ, Cohen MM Jr and Hennekam RCM: Syndromes of the Head and Neck. Oxford University Press, New York, p1179, 2001.

2. Van der Woude A: Fistula labii inferioris congenital and its association with cleft lip and palate. Am J Hum Genet 6: 254-266, 1954.

3. Gorlin RJ, Sedano HO and Cervenka J: Popliteal pterygium syndrome. A syndrome comprising cleft lip-palate, popliteal and intercrural pterygia, digital and genital anomalies. Pediatrics 41: 503-509, 1968 . 
4. Ghassibe M, Revencu N, Bayet B, Gillerot Y, Vanwijck R, Verellen-Dumoulin C and Vikkula M: Six families with van der Woude and/or popliteal pterygium syndrome: all with a mutation in the IRF6 gene. J Med Genet 41: E15-E19, 2004

5. Kondo S, Schutte BC, Richardson RJ, et al: Mutations in IRF6 cause Van der Woude and popliteal pterygium syndromes. Nat Genet 32: 285-289, 2002.

6. De Lima RL, Hoper SA, Ghassibe M, et al: Prevalence and nonrandom distribution of exonic mutations in interferon regulatory factor 6 in 307 families with Van der Woude syndrome and 37 families with popliteal pterygium syndrome. Genet Med 11: 241-247, 2009.

7. Tan EC, Lim EC, Yap SH, Lee ST, Cheng J, Por YC and Yeow V: Identification of IRF6 gene variants in three families with Van der Woude syndrome. Int J Mol Med: 747-751, 2008.

8. Zucchero TM, Cooper ME, Maher BS, et al: Interferon regulatory factor 6 (IRF6) gene variants and the risk of isolated cleft lip or palate. N Engl J Med 351: 769-780, 2004.

9. Diercks GR, Karnezis TT, Kent DT, Flores C, Su GH, Lee JH and Haddad J Jr: The association between interferon regulatory factor 6 (IRF6) and nonsyndromic cleft lip with or without cleft palate in a Honduran population. Laryngoscope 119: 1759-1764, 2009.

10. Cooper DN and Youssoufian $\mathrm{H}$ : The $\mathrm{CpG}$ dinucleotide and human genetic disease. Hum Genet 78: 151-155, 1988

11. Ingraham CR, Kinoshita A, Kondo S et al: Abnormal skin, limb and craniofacial morphogenesis in mice deficient for interferon regulatory factor 6 (IRF6). Nat Genet 38: 1335-1340, 2006.
12. Richardson RJ, Dixon J, Malhotra S, et al: IRF6 is a key determinant of the keratinocyte proliferation-differentiation switch. Nat Genet 38: 1329-1334, 2006.

13. Thomason HA, Zhou H, Kouwenhoven EN, et al: Cooperation between the transcription factors p63 and IRF6 is essential to prevent cleft palate in mice. J Clin Invest 120: 1561-1569, 2010.

14. Rahimov, F, Marazita ML, Visel A, et al: Disruption of an AP-2a binding site in an IRF6 enhancer is associated with cleft lip. Nat Genet 40: 1341-1347, 2008.

15. McGrath JA, Duijf PH, Doetsech V, et al: Hay-Wells syndrome is caused by heterozygous missense mutations in the SAM domain of p63. Hum Mol Genet 10: 221-229, 2001.

16. Milunsky JM, Maher TA, Zhao G, et al: TFAP2A mutations result in Branchio-oculo-facial syndrome. Am J Hum Genet 82: 1171-1177, 2008

17. Ali A, Singh SK and Raman R: Coding region of IRF6 may not be causal for Van der Woude syndrome in cases from India. Cleft Palate Craniofac J 46: 541-544, 2009.

18. Kollinen H, Wong FK, Rautio J, et al: Mapping of the second locus for the Van der Woude syndrome to chromosome 1p34. Eur J Hum Genet 9: 747-752, 2001.

19. Sertie AL, Sousa AV, Steman S, Pavanello RC and Passos-Bueno R: Linkage analysis in a large Brazilian family with Van der Woude syndrome suggests the existence of a susceptibility locus for cleft palate at 17p11.2-11. Am J Hum Genet 65: 433-440, 1999. 